\title{
Prion-like behavior of MAVS in RIG-I signaling
}

\author{
Eva Marie Y Moresco ${ }^{1}$, Diantha La Vine ${ }^{1}$, Bruce Beutler ${ }^{1}$ \\ ${ }^{1}$ Department of Genetics, The Scripps Research Institute, La Jolla, CA 92037, USA \\ Cell Research (2011) 21:1643-1645. doi:10.1038/cr.2011.155; published online 20 September 2011
}

Viral infection triggers the innate antiviral response in part through activation of RIG-I, a cytoplasmic sensor of 5'-triphosphorylated and uncapped single- or double-stranded RNA, which are not found among endogenous selfRNA. RIG-I signaling depends on the function of mitochondrial antiviral signaling (MAVS) protein, an adapter that links RIG-I, and the related RNA sensor MDA5, to events leading to transcriptional activation by NF- $\kappa B$ and IRF3. In contrast to the cytoplasmic localization of other innate immune signaling adapters, MAVS is positioned at mitochondrial membranes [1], and to a lesser extent at peroxisomal membranes [2], by virtue of a C-terminal transmembrane domain. For unknown reasons, mitochondrial localization is necessary for MAVS to propagate RIG-I signaling [1], except when MAVS is artificially dimerized [3]. MAVS-deficient mice fail to produce type I interferon in response to polyI:C stimulation and are highly susceptible to RNA virus infection, supporting an essential role for MAVS in innate antiviral immunity $[4,5]$.

Recently, the group of Zhijian Chen developed a cell free system that reconstitutes the complete RIG-I signaling pathway from 5'-triphosphorylated RNA (5'-pppRNA) to IRF3 activation [6]. Hou et al. have now used this powerful in vitro system to make the

Correspondence: Bruce Beutler

E-mail: bruce@scripps.edu remarkable discovery that MAVS forms prion-like aggregates to propagate RIGI signaling [7]. They mixed recombinant RIG-I, mitochondria, 5'-pppRNA, ATP, and K63 ubiquitin chains (K63-Ub4), or isolated mitochondria from cells infected with Sendai virus. When they analyzed MAVS from each of these preparations, they found that MAVS formed detergent- and protease-resistant aggregates larger in size than the $26 \mathrm{~S}$ proteasome. These aggregates formed in response to RIG-I activation, either by 5'-pppRNA and K63-Ub4, or by virus infection, and induced IRF3 activation. Using purified, recombinant MAVS lacking the transmembrane and prolinerich domains, it was shown that MAVS spontaneously formed large fiber-like polymers capable of activating IRF3 in the presence of cytosolic extracts, and remarkably, of rapidly inducing the formation of similar aggregates by endogenous MAVS on mitochondria from HEK293T cells. Mitochondria exposed to the recombinant MAVS fibers also became competent to activate IRF3. The prion-like ability of MAVS fibers to convert endogenous MAVS on the mitochondrial membrane to an aggregated form was solely dependent on the presence of the CARD domain (in both recombinant and endogenous proteins), whereas sequences C-terminal to the CARD domain (but excluding the transmembrane domain) were also necessary for IRF3 activation. Thus, Hou et al. establish an unprecedented paradigm for innate immune signaling: signal transduction by autocatalytic conformational conversion of an adapter.

Examples of phenotypic regulation by prion-induced changes in protein conformation remain relatively rare. To date, prion protein $(\mathrm{PrP})$, which causes the transmissible spongiform encephalopathies Creutzfeld-Jacob syndrome and bovine spongiform encephalopathy (mad cow disease), is the only known mammalian prion. Conformational changes of PrP, from a folded $\alpha$-helical structure to an infectious, aggregating, $\beta$ sheet-rich form, replicate through contact between the normal and prion conformers and lead to disease [8]. In Aplysia, a self-perpetuating conformer of cytoplasmic polyadenylation element-binding protein 1 (CPEB) supports a type of memory known as longterm facilitation [9]. By far the largest number of prions has been identified in $S$. cerevisiae and $P$. anserina. At least nine are known, most of which display multiple conformations associated with distinct, transmissible phenotypes [8].

There are several parallels between the properties of MAVS and known prion proteins. Most strikingly, aggregated MAVS had the ability to convert unaffected, mitochondrially localized MAVS molecules to the aggregated form, possibly through direct physical contact although this was not formally demonstrated. In addition, recombinant MAVS formed fiber-like polymers, similar in appearance to those formed by PrP, in a CARD domain-dependent manner. MAVS polymers were also 
resistant to protease and detergent treatments, features common to prions. Despite these similarities, MAVS aggregates did not stain with Congo red, a dye commonly used to label $\beta$ sheet-rich, insoluble aggregates called amyloids and formed by most prions. Consistent with this finding, the MAVS CARD domain appears to lack amyloid prion-forming domains that are enriched for polar glutamine and/or asparagine residues. Glutamines (and asparagines) are thought to hydrogen bond with each other to hold parallel amyloid $\beta$ sheets together, and to direct new monomers joining the amyloid filament to assume the same conformation as the previous molecules (i.e., replication) [10]. The three dimensional atomic structure of MAVS aggregates may prove to be non-amyloid; non-amyloid prions are known to exist [11] but their mechanisms of replication are much less well characterized.

Understanding how MAVS aggregates mediate RIG-I signaling will require answering how MAVS aggregation is initiated and how it is resolved in vivo. MAVS fiber formation in cells has not been observed, and may provide insight into the mechanism by which the prion-like conformation of MAVS differs from the inactive conformation, and how nucleation at the mitochondrial membrane occurs. Notably, a recent study demonstrated that the plasma membrane is the initial site of conversion of cellular PrP to the prion form [12], raising the possibility that MAVS conversion to the activated prion-like form also requires membrane association, in this case with mitochondria. Questions also remain regarding the role of MAVS-RIG-I interaction in the MAVS activation/aggregation process. One possible scenario is that physical interaction with activated RIG-I induces the conformational switch to the prion-like form of MAVS, which is subsequently propagated to other molecules of MAVS. Conversion of the cellular form of $\operatorname{PrP}\left(\operatorname{PrP}^{\mathrm{C}}\right)$ to the prion form $\left(\mathrm{PrP}^{\mathrm{Sc}}\right)$ entails binding of $\operatorname{PrP}^{\mathrm{C}}$ to an oligomer of $\mathrm{PrP}^{\mathrm{Sc}}$, a process called 'seeding'. Analogously, dimers or oligomers of RIG-I may seed the formation
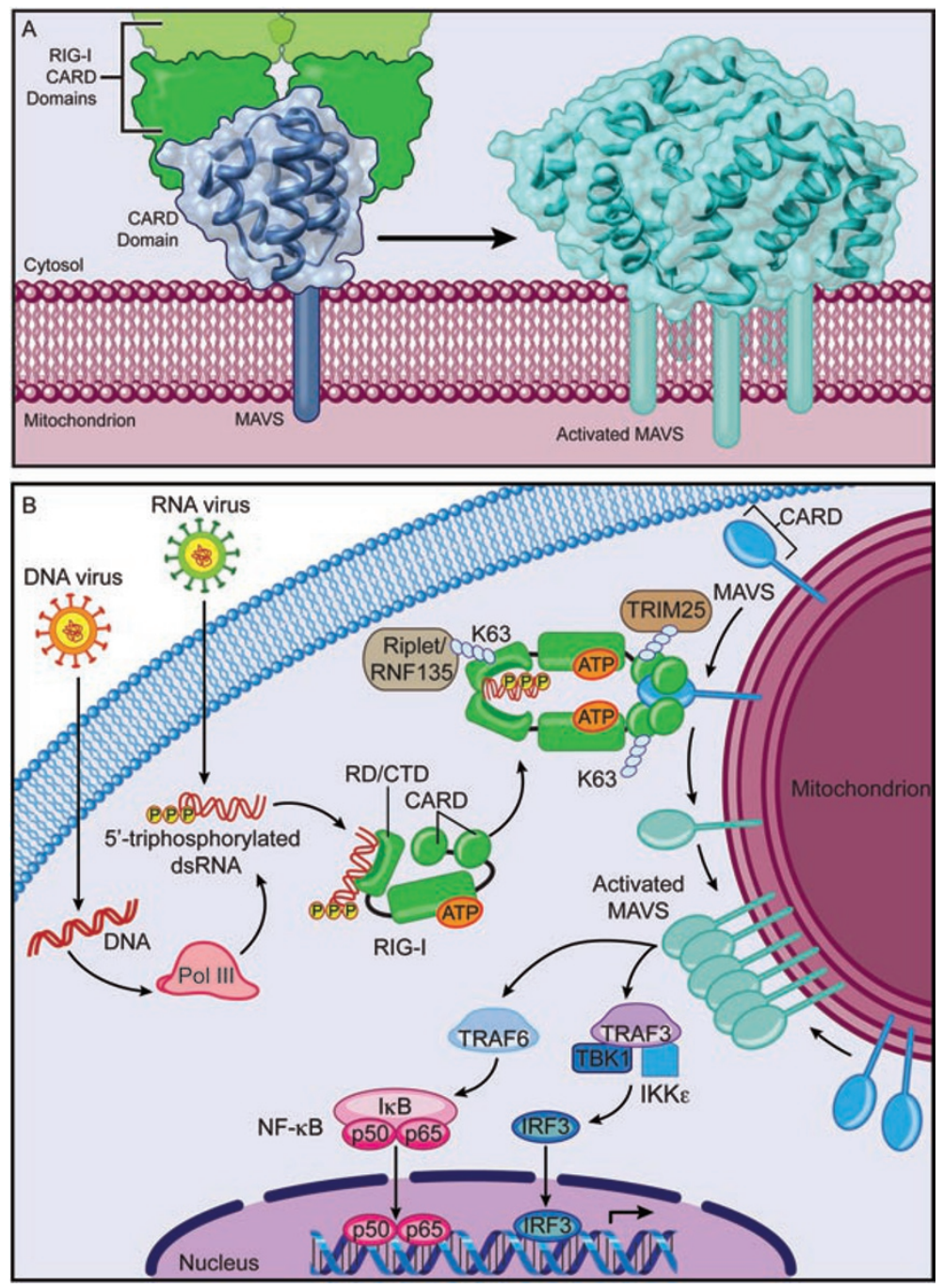

Figure 1 Hypothetical model of the switch to the prion-like form of MAVS. (A) The switch may be triggered by interactions between the non-prion CARD domain of MAVS (blue) and the CARD domains of dimers or oligomers of RIG-I (green), which induce a conformational alteration promoting the prion-like state of MAVS (teal). Once converted to this state, activated MAVS forms aggregates, possibly in a left-handed helix formation as has been observed in the Myddosome, which also interfaces with TRAF3 and TRAF6 to transduce signaling. Model of MAVS CARD domain (Protein Data Bank Accession 2VGQ) was generated using UCSF Chimera. (B) RIG-I signaling. 5'-triphosphorylated RNA from DNA or RNA viruses binds to the C-terminal domain (RD/CTD) of RIG-I, causing it to release autoinhibitory interactions. Full activation of RIG-I requires binding to unanchored K63 polyubiquitin chains (K63), as well as conjugation to K63 polyubiquitin chains by TRIM25 and Riplet/RNF135. CARD-CARD interactions between RIG-I and MAVS lead to MAVS activation, which involves a switch to the prion-like conformation and aggregation into large polymers. These polymers activate TRAF6 and TRAF3, leading to NF- $\kappa$ B- and IRF3dependent gene transcription. 
of MAVS prion-like aggregates (Figure 1). Generation of the first MAVS-prion template by RIG-I would ensure that MAVS activation/aggregation only occurs in response to viral infection or other RIG-I activators. Hou et al. note that the CARD domains of RIG-I and MDA5 share little sequence homology with that of MAVS, suggesting the possibility that the conformationally distinct RIG-I CARD domain could induce alterations to the MAVS CARD structure that promote the prion-like conformation. They also demonstrated that geldanamycin, an inhibitor of Hsp90, blocks the formation of MAVS aggregates, suggesting that Hsp90 may be required for assembly of the aggregates; a caveat of this finding is that the action of geldanamycin was not shown to be restricted to Hsp90.

The removal of MAVS aggregates is presumably required to halt signaling, and conversely, persistence of the aggregates may result in unmitigated signaling leading to autoimmunity. PCBP2 has been reported to recruit the E3 ligase AIP4 to MAVS, leading to MAVS ubiquitination and subsequent degradation by the proteasome [13]. However, the degradation of MAVS aggregates by this mechanism has not been investigated. It seems unlikely that MAVS-prions are inactivated by dilution, which occurs in yeast by cell division and affects the persistence of yeast prions. Further studies on prion degradation and its regulation, about which little is known, may shed light on the mechanism of removal of MAVS aggregates.

One of the most intriguing questions raised by the findings of Hou et $a l$. is how downstream signaling is propagated by aggregates of MAVS. The signaling events downstream from MAVS, namely TRAF6 activation leading to IKK complex formation and $\mathrm{NF}-\kappa \mathrm{B}$ activation, as well as TRAF3 activation leading to TBK1-dependent IRF3 activation, are identical to those downstream from MyD88, an adapter for the Toll-like receptors (TLRs; except TLR3) and interleukin-1 and -18 receptors (IL-1R/18R). It seems plausible that the MyD88 and MAVS adapter complexes, both of which signal to TRAF6 and TRAF3, bear some structural similarity, at least at the interface with these TRAFs, that permit them to interact with and/or activate the TRAFs. Recently, the crystal structure of the signaling complex formed by the death domains (DDs) of MyD88, IRAK4, and IRAK2, termed the Myddosome, was reported [14]. Like the isolated CARD domain of MAVS [15], the DD of MyD88, IRAK4, and IRAK2 folds into a six-helix bundle; six MyD88, four IRAK4, and four IRAK2 DDs are arranged in a left-handed helix in the Myddosome, triggering downstream signaling. Assembly of the Myddosome, with MyD88 molecules oligomerizing to form the initial 'seed' for complex formation, is reminiscent of the seeding of prion polymers. An interesting possibility is that, seeded by a complex of RIG-I CARD domains, the six-helix bundle CARD domain of MAVS might also form left-handed helical fibers or aggregates to activate TRAF6 and TRAF3 (Figure 1).

The idea that immune signaling depends upon autocatalytic changes in protein conformation unites two very different fields and makes us wonder whether, as with prions, specific mutations of MAVS or related proteins can cause spontaneous assembly, altering the propensity to develop disease or causing it outright.

\section{References}

1 Seth RB, Sun L, Ea CK, Chen ZJ. Identification and characterization of MAVS, a mitochondrial antiviral signaling protein that activates NF-kappaB and IRF 3. Cell 2005; 122:669-682.

2 Dixit E, Boulant S, Zhang Y, et al. Peroxisomes are signaling platforms for antiviral innate immunity. Cell 2010; 141:668-681.

3 Tang ED, Wang CY. MAVS self-associ- ation mediates antiviral innate immune signaling. J Virol 2009; 83:3420-3428.

4 Sun Q, Sun L, Liu HH, et al. The specific and essential role of MAVS in antiviral innate immune responses. Immunity 2006; 24:633-642.

5 Kumar H, Kawai T, Kato H, et al. Essential role of IPS-1 in innate immune responses against RNA viruses. $J$ Exp Med 2006; 203:1795-1803.

6 Zeng W, Sun L, Jiang X, et al. Reconstitution of the RIG-I pathway reveals a signaling role of unanchored polyubiquitin chains in innate immunity. Cell 2010; 141:315-330.

7 Hou F, Sun L, Zheng H, Skaug B, Jiang QX, Chen ZJ. MAVS forms functional prion-like aggregates to activate and propagate antiviral innate immune response. Cell 2011; 146:448-461.

8 Tuite MF, Serio TR. The prion hypothesis: from biological anomaly to basic regulatory mechanism. Nat Rev $\mathrm{Mol}$ Cell Biol 2010; 11:823-833.

9 Si K, Choi YB, White-Grindley E, Majumdar A, Kandel ER. Aplysia CPEB can form prion-like multimers in sensory neurons that contribute to longterm facilitation. Cell 2010; 140:421435.

10 Wickner RB, Edskes HK, Kryndushkin D, McGlinchey R, Bateman D, Kelly A. Prion diseases of yeast: Amyloid structure and biology. Semin Cell Dev Biol 2011 February 21; doi:10.1016/j. semcdb.2011.02.021

11 Roberts BT, Wickner RB. Heritable activity: a prion that propagates by covalent autoactivation. Genes Dev 2003; 17:2083-2087.

12 Goold R, Rabbanian S, Sutton L, et al. Rapid cell-surface prion protein conversion revealed using a novel cell system. Nat Commun 2011; 2:281.

13 You F, Sun H, Zhou X, et al. PCBP2 mediates degradation of the adaptor MAVS via the HECT ubiquitin ligase AIP4. Nat Immunol 2009; 10:13001308.

14 Lin SC, Lo YC, Wu H. Helical assembly in the MyD88-IRAK4-IRAK2 complex in TLR/IL-1R signalling. $\mathrm{Na}$ ture 2010; 465:885-890.

15 Potter JA, Randall RE, Taylor GL. Crystal structure of human IPS-1/ MAVS/VISA/Cardif caspase activation recruitment domain. BMC Struct Biol 2008; 8:11. 